TRABAJO ORIGINAL

\title{
EFECTO ANTIVASCULAR DE VINCRISTINA EN TUMOR VENÉREO TRANSMISIBLE DEL CANINO
}

\author{
ANTIVASCULAR EFFECT OF VINCRISTINE SURPHATE ON \\ CANINE TRANSMISSIBLE VENEAL TUMOR
}

\author{
GonZalez, C.M. ${ }^{1}$; Cepeda, R. ${ }^{2} ;$ Flores, $^{3}{ }^{3}$; Cattaneo, G. ${ }^{3}$; Contreras, Francisco
}

\author{
ABSTRACT
}

\begin{abstract}
Among the more investigated antineoplastic therapeutic options, the anti-angiogenic therapy has no important collateral effects for the patient. This therapy targets the factors implied in the progress of the angiogenic cascade, to impede the vascular support of tumoral tissue, increasing the apoptosis of the neoplastic cells.

In this study the changes of the vascularization of canine venereal transmissible tumor (CTVT) were studied, both in the progressive phase of growth and during regression induced by treatment with vincristine sulphate (VCR). This antineoplastic drug apart from its cytostatic effect on tumors, it has demonstrated direct antivascular effect.

Histopathologically with Papanicolau stain, was observed that after the treatment, the number of blood vessels diminished significantly $(p<0.05)$. On the other hand it was determined, by computer assisted morphometry that the area occupied by the erythrocyte was significantly smaller $(p<0.05)$ in regressive CTVT. Also, the total average area occupied by blood vessels was significantly smaller $(p<0.05)$ in regression of CTVT growth induced with VCR.
\end{abstract}

Key words: Transmisible venereal tumor, Vascularization, Vincristine.

\section{INTRODUCCIÓN}

El TVT se caracteriza, en su presentación clínica, por su aspecto hemorrágico, asociado a una extensa irrigación sanguínea, que le sirve de sostén para recibir los componentes que le permiten desarrollar su acelerado metabolismo. Esto sugiere, que este tumor sería un excelente modelo para el estudio de angiogénesis tumoral y de su comportamiento frente al tratamiento con drogas antineoplásicas, convencionales y antiangiogénicas.

En este estudio se caracterizarán los cambios que presenta el lecho vascular tumoral del TVT, tanto en su fase de crecimiento progresivo como durante la regresión inducida por el tratamiento con sulfato de vincristina (VCR), droga antineoplásica que, pertenece a Los alcaloides de la vinca (VA) que actúan como citostáticos, suprimiendo la dinámica de los microtúbulos (MT) (Jordan y Wilson, 2004), a través de la unión a la proteína microtubular tubulina en la fase $\mathrm{M}$ del ciclo celular, lo que impide la formación del huso mitótico, (Adams et al., 1981; González et al., 2002) y por consiguiente, inhibe la proliferación de las células tumorales debido al bloqueo de la mitosis (Brown et al., 1980; Booth, 1994; Jeglum, 1994; González et al., 2002).

Se ha indicado que la VCR también induciría la apoptosis o "muerte programada" de las células de TVT (Torres, 1999), pues se ha observado que luego de la quimioterapia, se produce un incremento en el

1 Fac. Cs. Veterinarias y Pecuarias U. de Chile Dpto. Patología Animal.

2 Fac. Cs. Veterinarias y Pecuarias U. De Chile Dpto. Ciencias Básicas.

3 Fac. Cs. Veterinarias y Pecuarias U. De Chile Dpto. Ciencias Clínicas. Financiado por Proyecto FONDECYT 1020980 . 
recuento de células con características apoptóticas en la fase regresiva tumoral (Booth, 1994; Reyes, 2004), como la marginación de la cromatina nuclear, la condensación del núcleo, el recogimiento de citoplasma y cuerpos apoptóticos en espacio extracelular y dentro de células fagocíticas (Reyes, 2004). Existen trabajos que relacionan el efecto pro-apoptótico de la VCR con el cáncer mamario (Aoudjit y Vuori, 2001), el osteosarcoma (Seki et al., 2000) y otros tumores humanos o líneas de células tumorales (Belhoussine et al., 1999) como la leucemia infantil, en la que se describió la activación de las caspasas 9 y 3 (Srivastava et al., 1998; Groninger et al., 2002), que sería regulada a través de la generación temprana de radicales libres (especies reactivas de oxígeno), por lo que estaría implicada la vía mitocondrial o intrínseca del ciclo apoptótico (Groninger et al., 2002).

Recientemente, se ha descrito otra acción antitumoral de los agentes antitubulínicos, demostrando una actividad antivascular ejercida directamente sobre el endotelio tumoral en proliferación, ocluyendo o destruyendo vasos sanguíneos pre-existentes (Natsume et al., 2003).

\section{MATERIALES Y MÉTODOS}

\section{Muestras biológicas:}

Se utilizaron biopsias de TVT, de tumores de ubicación genital de 10 caninos adultos de entre 1 a 10 años de edad, sin distinción de sexo ni raza, los que fueron diagnosticados clínicamente, en el Servicio de Cirugía de la Facultad de Ciencias Veterinarias y Pecuarias de la Universidad de Chile. Los pacientes fueron sometidos a quimioterapia, utilizando VCR (Vincristina Sulfato ${ }^{\mathrm{R}}$ Pharmachemie B.D. Holland), en dosis única de $0,03 \mathrm{mg} / \mathrm{kg}$, diluida en agua bidestilada, administrada vía endovenosa, para inducir regresión tumoral (Lombardi, 1995).

El diagnostico de T.V.T se confirmó mediante extendidos celulares sobre portaobjetos, mediante impronta o raspado de la masa tumoral. Estas fueron fijadas en metanol, teñidas con colorante Giemsa durante 10 minutos, lavadas con agua destilada y secadas al medio ambiente (Duarte, 2002).

Se obtuvieron muestras de tejido neoplásico, mediante biopsias de todos los individuos, antes y después de la quimioterapia, las que se fijaron en formalina al $10 \%$ por 24 hrs (Duarte, 2002). Éstas se procesaron de acuerdo a las técnicas convencionales para tejidos incluidos en parafina (Lopez et al, 1985), obteniendo cinco cortes seriados de $5 \mu \mathrm{m}$ de grosor, con micrótomo de rotación. Los cortes seriados obtenidos, fueron sometidos a tinciones con Hematoxilina/Eosina, Papanicolau, PAS y van Gieson.

\section{Analisis morfométrico:}

Para comparar el lecho vascular de las muestras en estudio, se realizó el análisis morfométrico de eritrocitos y de vasos sanguíneos presentes, en biopsias de fases progresiva y regresiva del TVT. Se digitalizaron las imágenes de 5 campos de 200X elegidos al azar dentro de cada caso, con una resolución de 512 X 480 pixeles, mediante una videocámara digital, montada en un microscopio óptico científico, y conectada a un computador al que se le había incorporado el "software" morfométrico. De este modo, se marcó y midió, en micrómetros cuadrados $\left(\mu \mathrm{m}^{2}\right)$, el área total abarcada por los eritrocitos y por los vasos sanguíneos, ubicados en el interior de cada uno de los cinco campos para cada caso, en fase tumoral progresiva y regresiva.

Así, como una forma indirecta de estimación del grado de vascularización del tejido tumoral, una vez determinada el área ocupada por los eritrocitos, se obtuvo la sumatoria de los cinco campos para cada uno de los casos y luego, la sumatoria de todos los casos para cada fase tumoral, para estimar, mediante análisis estadístico, la variación que presentaba el área total ocupada por eritrocitos en cada caso, antes y después de instaurada la fase regresiva tumoral. Los valores de área en cada campo fueron determinados mediante el reconocimiento morfométrico computacional de los eritrocitos presentes, a través del programa ImageProPlus y con ayuda de tinción de Papaniculoau, en que los eritrocitos se tiñen específicamente de un color anaranjado característico, logrando así ser registrados por el software morfométrico.

Por otro lado, como una de estimación más directa del grado de vascularización del tejido tumoral, se estudió el área ocupada directamente por vasos sanguíneos visibles en los campos de TVT, de fase progresiva y regresiva. Se obtuvo la sumatoria de los cinco campos para cada uno de los casos y luego, la sumatoria de todos los casos, para cada fase tumoral, con el fin de compararlos mediante análisis estadístico y estimar la variación que presentaba el área total ocupada por los vasos sanguíneos en cada caso, antes y después de instaurada la fase regresiva tumoral mediante la aplicación de tratamiento quimioterápico con VCR. Los valores se determinaron mediante medición en $\mu \mathrm{m}^{2}$ del área ocupada por los vasos sanguíneos intratumorales presentes, con ayuda 
del programa morfométrico computacional ImageProPlus ${ }^{\circledR}$. Los vasos sanguíneos fueron previamente marcados, en forma manual, con ayuda del programa AdobePhotoShop ${ }^{\circledR}$.

Finalmente, se obtuvo la sumatoria del número de vasos presentes en cada uno de los cinco campos en cada uno de los casos, tanto para fase progresiva como regresiva y se procedió a dividir el área vascular total de cada uno de los casos por el número de vasos sanguíneos presentes en los cinco campos correspondientes, con el fin de estimar si existían diferencias en el área ocupada en promedio por los vasos sanguíneos presentes en cada fase de crecimiento tumoral.

\section{Análisis estadístico:}

Los valores de las sumatorias de las áreas ocupadas por eritrocitos y por vasos sanguíneos, obtenidas del análisis morfométrico, y de los productos de las áreas vasculares por el número de vasos presentes, fueron comparados en relación a las fases tumorales (progresiva y regresiva), mediante el empleo de pruebas de $t$ para muestras asociadas, suponiendo varianzas iguales (Snedecor y Cochran, 1980), para determinar si las diferencias en la estimación del lecho vacular eran estadísticamente significativas.

\section{RESULTADOS}

\section{Características histopatológicas del lecho vascular del TVT}

Previo al tratamiento con VCR, en cortes teñidos con PAP, H-E, PAS y van Gieson, se observó la presencia de abundantes vasos sanguíneos. Los vasos sanguíneos correspondían, principalmente a vasos de pequeño y de mediano calibre, ubicados entre las células tumorales, en el estroma. correspondiendo principalmente a arteriolas, metarteriolas, vénulas, venas de pequeño calibre y capilares, sin un claro predominio de uno sobre otro. En algunos casos, se observó amplias zonas de hemorragia en el tejido tumoral..

La pared de las arteriolas presentaba una a dos capas de tejido muscular liso, que por medio de la tinción PAP, se observaron de diferentes tonalidades, desde un color verde azulado claro o rosado. En general, estos vasos se observaron dilatados, conteniendo una gran cantidad de glóbulos rojos en su interior, que teñían de un color anaranjado con PAP (Figura 1).
Luego del tratamiento con una dosis de VCR, los vasos sanguíneos predominantes continuaban siendo de pequeño calibre, pero no se encontraron distendidos y la presencia de glóbulos rojos en su interior fue escasa (Figura 2). La cantidad de vasos sanguíneos y de eritrocitos presentes en el lecho vascular, en las muestras posteriores al tratamiento, fueron notoriamente menores que en las muestras tomadas previo al tratamiento con VCR (Figura 3).

\section{ANÁLISIS MORFOMÉTRICO}

\section{Estimación del grado de vascularización} del TVT a través de la detección de eritrocitos

Como una estimación indirecta del grado de vascularización del tejido tumoral, se determinó el área ocupada por los eritrocitos presentes en cinco campos de 200X de TVT, en cada uno de los casos estudiados, tanto para la fase progresiva de crecimiento tumoral, como luego de iniciada la regresión mediante la aplicación de tratamiento quimioterápico con VCR. Posteriormente, se obtuvo la sumatoria de los cinco campos para cada uno de los casos y la sumatoria total de todos los casos para cada fase tumoral, con el fin de compararlos y estimar la variación que presentaba el área total ocupada por eritrocitos en cada caso, antes y después de instaurada la fase regresiva tumoral, mediante el análisis estadístico con prueba de $t$, suponiendo varianzas iguales.

En la tabla 1 se resumen los datos de las sumatorias de las áreas ocupadas por eritrocitos $\left(\mu \mathrm{m}^{2}\right)$ de cinco campos de 200X, para cada caso de TVT canino, donde se observa que el promedio total de los casos en fase progresiva es significativamente mayor $(\mathrm{p} \leq 0.05)$, con un valor de $12.062,11 \pm 10.671,13$ $\mu \mathrm{m}^{2}$ en comparación con $1.199,87 \pm 1.850,09 \mu^{2}$, obtenido para la fase regresiva.

En la figura 4 se observa que en cada uno de los diez casos estudiados, las sumatorias de área ocupada por eritrocitos en los cinco campos (200X) son mayores en los casos de TVT progresivo, previo al tratamiento, en comparación con las de fase regresiva inducida con VCR. En la fase regresiva de los casos 6 y 10 , no se observó la presencia de eritrocitos.

6.2.2. Estimación del grado de vascularización del TVT a través del área vascular total y el área promedio ocupada por los vasos intratumorales

Por otro lado, como una forma más directa de ẹstimación del grado de vascularización del tejido tumoral, se determinó el área total ocupada por vasos sanguíneos 


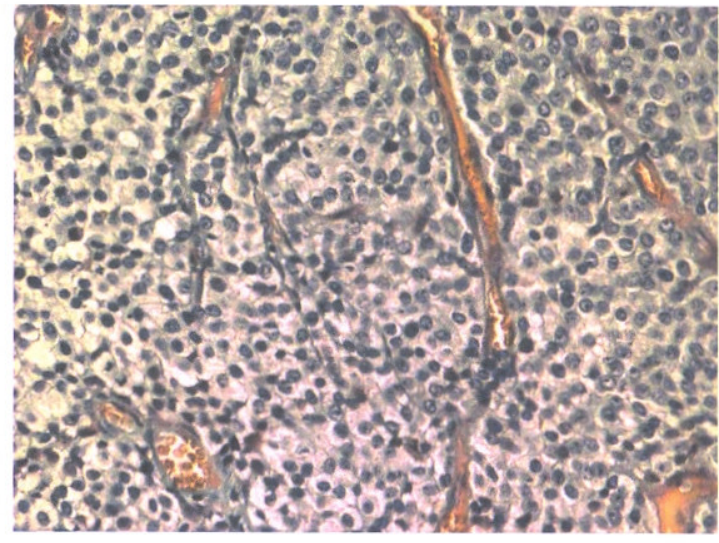

Figura 1, TVT progresivo, tinción Papanicolau, 200X. Demostración de vaso sanguíneo conteniendo eritrocitos de color anaranjado.

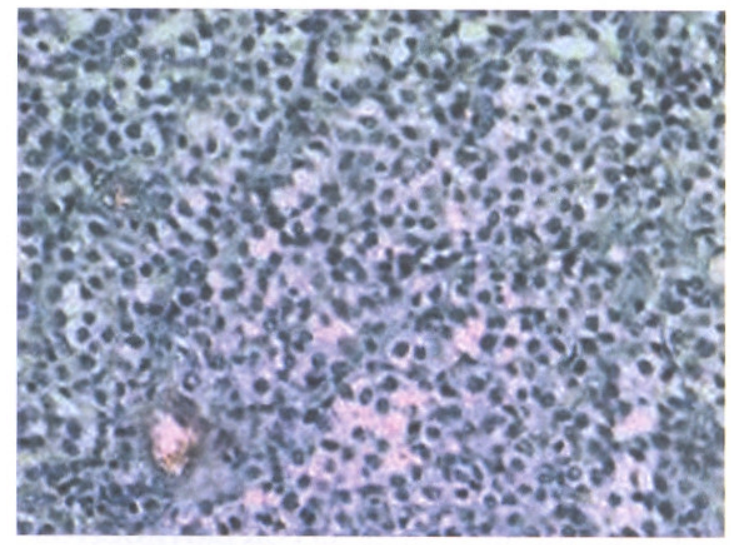

Figura 2, TVT regresivo, tinción Papanicolau, 200X. Escasos vasos sanguíneos conteniendo eritrocitos.

\begin{tabular}{lccccc}
\hline & & \multicolumn{4}{c}{ Diámetro } \\
\cline { 3 - 6 } Fase tumoral & & $00,01-10,00 \mu \mathrm{m}$ & $10,01-20,00 \mu \mathrm{m}$ & $20,01-30,00 \mu \mathrm{m}$ & $30,01-40,00 \mu \mathrm{m}$ \\
\hline Progresivo & PROM & 0,58 & 0,41 & 0,06 & 0,03 \\
& DS & 0,26 & 0,21 & 0,08 & 0,09 \\
\hline Regresivo & PROM & 0,72 & 0,19 & 0,00 & 0,00 \\
& DS & 0,38 & 0,29 & 0,00 & 0,0 \\
\hline
\end{tabular}

FIGURA 3

DISTRIBUCIÓN DE VASOS SANGUÍNEOS SEGÚN DIÁMETRO EN TVT PROGRESIVO Y REGRESIVO

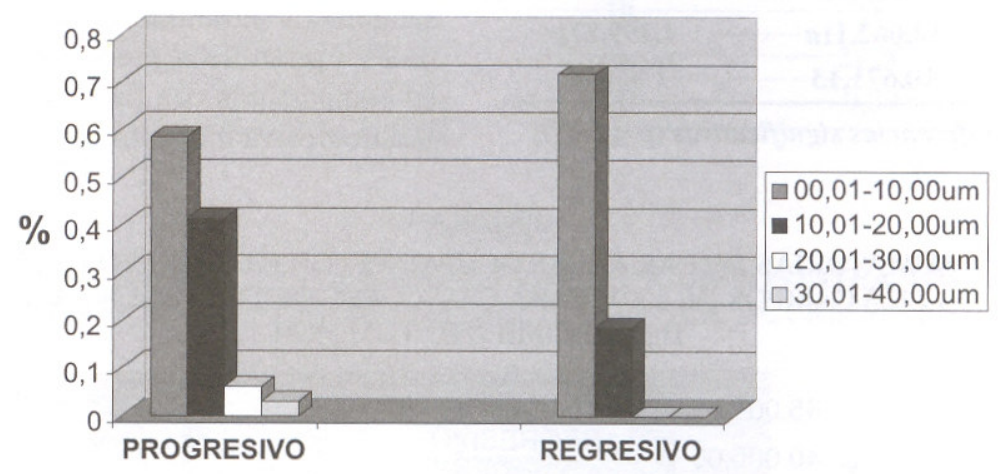

de diferente tipo presentes en cinco campos de 200X de TVT, para cada uno de los casos estudiados, tanto en fase progresiva de crecimiento como luego de iniciada la regresión mediante la aplicación de tratamiento quimioterápico con VCR. Posteriormente se obtuvo la sumatoria de los cinco campos para cada uno de los casos y la sumatoria total de todos los casos en cada fase tumoral, con el fin de compararlos y estimar la variación que presentaba el área total ocupada por vasos sanguíneos en cada caso, antes y después de instaurada la fase regresiva tumoral mediante el análisis estadístico con prueba de $t$, suponiendo varianzas iguales.

En la Tabla 2 se resumen los datos de las sumatorias de las áreas ocupadas por los vasos sanguíneos intratumorales $\left(\mu \mathrm{m}^{2}\right)$ en cinco campos $(200 \mathrm{X})$ para cada caso de TVT canino, según fase de crecimiento tumoral. El promedio total de los casos en fase progresiva es significativamente mayor $(\mathrm{p} \leq 0.05)$, con un valor de $4.096,55 \pm 1.391,80 \mu \mathrm{m}^{2}$ en comparación con un 616,42 $\pm 459,71 \mu \mathrm{m}^{2}$ obtenido para la fase regresiva. 
En la figura 5 se observa que en cada uno de los diez casos estudiados, las sumatorias de los cinco campos (200X) de área ocupada por los vasos sanguíneos intratumorales son mayores en los casos de TVT progresivo, previo al tratamiento con VCR, en comparación con los casos correspondientes en fase regresiva inducida con VCR. En el caso 10 no se observó la presencia de vasos sanguíneos en el parénquima tumoral

TABLA 1

DISTRIBUCIÓN DE LOS SUMATORIAS DE LAS ÁREAS OCUPADAS POR ERITROCITOS $\left(\mu \mathrm{m}^{2}\right)$ EN CINCO CAMPOS DE $200 X$, PARA PARA CADA CASO DE TVT CANINO, SEGÚN FASE DE CRECIMIENTO TUMORAL

\begin{tabular}{ccc}
\hline & \multicolumn{2}{c}{ Fase crecimiento tumoral } \\
\cline { 2 - 3 } Caso & Progresivo & Regresivo \\
\hline $\mathbf{1}$ & $8.903,23$ & $1.868,35$ \\
\hline $\mathbf{2}$ & $10.317,44$ & 315,70 \\
\hline $\mathbf{3}$ & $4.831,75$ & 34,40 \\
\hline $\mathbf{4}$ & $7.109,08$ & 918,10 \\
\hline $\mathbf{5}$ & $16.304,22$ & 398,16 \\
\hline $\mathbf{6}$ & $6.485,44$ & 0,00 \\
\hline $\mathbf{7}$ & $10.393,72$ & 576,18 \\
\hline $\mathbf{8}$ & $12.226,21$ & $1.802,82$ \\
\hline $\mathbf{9}$ & $40.526,35$ & $6.085,01$ \\
\hline $\mathbf{1 0}$ & $\mathbf{3 . 5 2 3 , 6 5}$ & 0,00 \\
\hline Promedio & $\mathbf{1 2 . 0 6 2 , 1 1 a}$ & $\mathbf{1 . 1 9 9 , 8 7 b}$ \\
\hline DS & $\mathbf{1 0 . 6 7 1 , 1 3}$ & $\mathbf{1 . 8 5 0 , 0 9}$ \\
\hline
\end{tabular}

a y $b$ representan diferencias significativas $(p \leq 0.05)$.
A continuación, obtuvo la sumatoria del número de vasos presentes en cada uno de los casos, tanto para fase progresiva como regresiva, enconitrando que en la mayoría de los casos disminuía el número de vasos presentes, luego de instaurada la fase regresiva después del tratamiento con VCR. En la la tabla 3 se resumen los datos del número de vasos sanguíneos, para cada caso de TVT canino, según fase de crecimiento tumoral.

En seguida, con la finalidad de estimar si existían diferencias asociadas al área promedio ocupada por los vasos sanguíneos presentes en cada fase de crecimiento tumoral, se calculó la división del área vascular total de cada uno de los casos por el número de vasos sanguíneos correspondientes, como se demuestra en la tabla 4 . En la tabla 5 se resumen los datos de los productos obtenidos. En la mayoría de los casos la división obtenida en ambas fases es significativamente diferente, con excepción de los inmdividuos 5 y 7 , en que la diferencia tiende a ser menor. Además se observa que, en relación al promedio de estos productos, los vasos de la fase progresiva ocupan, en promedio, un área mayor, con un valor de $218.14 \pm 86.35 \mu \mathrm{m}^{2}$ en comparación con un $76.27 \pm 65.01 \mu \mathrm{m}^{2}$, obtenido para los de fase regresiva, confirmando estadísticamente la existencia de una diferencia significativa ( $\mathrm{p} \leq 0.05)$.

En la figura se observa que, en la mayoría de los casos estudiados, el producto de las áreas vasculares totales $\left(\mu \mathrm{m}^{2}\right)$ por el número de vasos sanguíneos intratumorales, es mayor en los casos de TVT progresivo, previo al tratamiento con VCR, en comparación con los casos correspondientes en fase regresiva inducida con VCR.

FIGURA 4

SUMATORIAS DE LAS ÁREAS OCUPADAS POR ERITROCITOS $\left(\mathrm{MM}^{2}\right)$ EN 5 CAMPOS DE 200X, PARA CADA CASO DE TVT SEGÚN FASE DE CRECIMIENTO TUMORAL

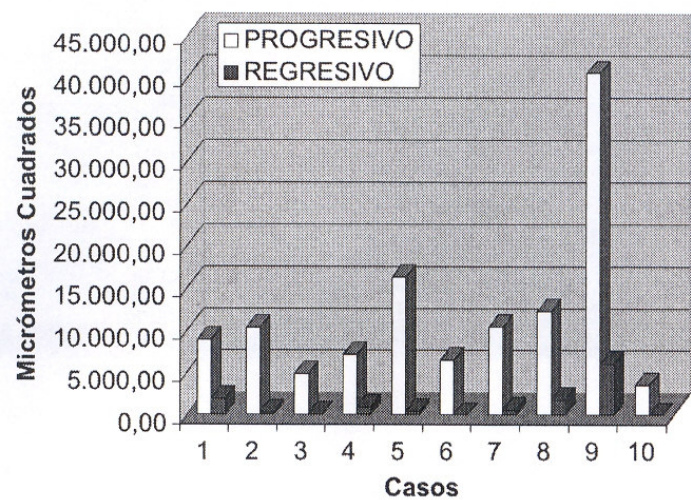


TABLA 2

DISTRIBUCIÓN DE LA SUMATORIA DE LAS ÁREAS DE CINCO CAMPOS DE 200X OCUPADAS POR LOS VASOS SANGUÍNEOS INTRATUMORALES $\left(\mu \mathrm{m}^{2}\right)$, PARA CADA CASO DE TVT CANINO, SEGÚN FASE DE CRECIMIENTO TUMORAL

\begin{tabular}{ccc}
\hline & \multicolumn{2}{c}{ Fase crecimiento tumoral } \\
\cline { 2 - 3 } Caso & Progresivo & Regresivo \\
\hline $\mathbf{1}$ & $4.355,90$ & 332,59 \\
\hline $\mathbf{2}$ & $3.297,32$ & 372,01 \\
\hline $\mathbf{3}$ & $4.593,75$ & 178,66 \\
\hline $\mathbf{4}$ & $3.712,78$ & 448,38 \\
\hline $\mathbf{5}$ & $7.269,35$ & $1.504,83$ \\
\hline $\mathbf{6}$ & $1.993,00$ & 671,01 \\
\hline $\mathbf{7}$ & $2.808,23$ & 574,17 \\
\hline $\mathbf{8}$ & $4.254,05$ & 982,67 \\
\hline $\mathbf{9}$ & $4.478,69$ & $1.099,86$ \\
\hline $\mathbf{1 0}$ & $4.202,40$ & 0,00 \\
\hline Promedio & $\mathbf{4 . 0 9 6 , 5 5} \boldsymbol{a}$ & $\mathbf{6 1 6 , 4 2} \boldsymbol{b}$ \\
\hline DS & $\mathbf{1 . 3 9 1 , 8 0}$ & $\mathbf{4 5 9 , 7 1}$ \\
\hline
\end{tabular}

a y $b$ representan diferencias significativas $(p \leq 0.05)$.

\section{DISCUSIÓN}

Características vasculares y cuantificación del lecho vascular del tvt, en fase progresiva y regresiva

El estroma tumoral del TVT en etapa progresiva se observó muy vascularizado, coincidiendo con los descrito por Rogers (1997), González et al. (2000) y Tella et al. (2004). Histológicamente, los vasos observados correspondían, en su mayoría, a arteriolas y metarteriolas, lo que se asemeja a lo observado por Ramos (2004), quién análizo el área endotelial del TVT mediante técnica inmunohistoquímica, utilizando como marcador de células endoteliales anticuerpos contra el factor de von Willebrand.

Luego del tratamiento con una dosis de VCR, los tipos de vasos sanguíneos presentes en él y su

TABLA 3

NÚMERO TOTAL DE VASOS SANGUÍNEOS PRESENTES EN CINCO CAMPOS DE 200X DE TVT CANINO, PARA CADA CASO SEGÚN FASE DE CRECIMIENTO TUMORAL

\begin{tabular}{ccc}
\hline & \multicolumn{2}{c}{ Fase crecimiento tumoral } \\
\cline { 2 - 3 } Caso & Progresivo & Regresivo \\
\hline $\mathbf{1}$ & 24 & 8 \\
\hline $\mathbf{2}$ & 8 & 2 \\
\hline $\mathbf{3}$ & 23 & 12 \\
\hline $\mathbf{4}$ & 20 & 14 \\
\hline $\mathbf{5}$ & 36 & 8 \\
\hline $\mathbf{6}$ & 8 & 13 \\
\hline $\mathbf{7}$ & 30 & 7 \\
\hline $\mathbf{8}$ & 30 & 12 \\
\hline $\mathbf{9}$ & 19 & 13 \\
\hline $\mathbf{1 0}$ & 15 & 0 \\
\hline Promedio & 21,30 & 8,90 \\
\hline SD & 9,30 & 4,84 \\
\hline
\end{tabular}

FIGURA 5

SUMATORIAS DE LAS ÁREAS OCUPADOS POR VASOS SANGUÍNEOS INTRATUMORALES $\left(\mu \mathrm{m}^{2}\right)$ EN 5 CAMPOS DE 200X PARA CADA CASO DE TVT, SEGÚN FASE DE CRECIMIENTO TUMORAL

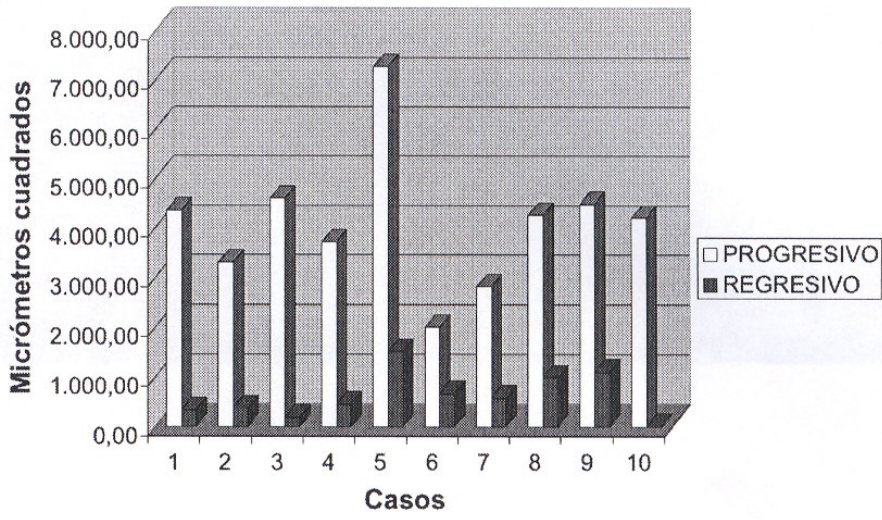


TABLA 4

DIVISIÓN DEL ÁREA VASCULAR TOTAL DE CADA CASO POR EL NÚMERO DE VASOS SANGUÍNEOS PRESENTE EN LOS CINCO CAMPOS CORRESPONDIENTES

\begin{tabular}{ccccccccc}
\hline & \multicolumn{3}{c}{ Fase Progresiva } & & \multicolumn{3}{c}{ Fase Regresiva } \\
\cline { 2 - 3 } Caso & Área Total & Vasos & Producto & & Área Total & Vasos & Producto \\
\hline $\mathbf{1}$ & $4.355,90$ & 24 & $\mathbf{1 8 1 . 5 0}$ & & 332,59 & 8 & $\mathbf{4 1 . 5 7}$ \\
\hline $\mathbf{2}$ & $3.297,32$ & 8 & $\mathbf{4 1 2 . 1 7}$ & & 372,01 & 2 & $\mathbf{1 8 6 . 0 1}$ \\
\hline $\mathbf{3}$ & $4.593,75$ & 23 & $\mathbf{1 9 9 . 7 3}$ & & 178,66 & 12 & $\mathbf{1 4 . 8 8}$ \\
\hline $\mathbf{4}$ & $3.712,78$ & 20 & $\mathbf{1 8 5 . 6 4}$ & & 448,38 & 14 & $\mathbf{3 2 . 0 3}$ \\
\hline $\mathbf{5}$ & $7.269,35$ & 36 & $\mathbf{2 0 1 . 9 3}$ & & $1.504,83$ & 8 & $\mathbf{1 8 8 . 1}$ \\
\hline $\mathbf{6}$ & $1.993,00$ & 8 & $\mathbf{2 4 9 . 1 3}$ & & 671,01 & 13 & $\mathbf{5 1 . 6 2}$ \\
\hline $\mathbf{7}$ & $2.808,23$ & 30 & $\mathbf{9 3 . 6 1}$ & & 574,17 & 7 & $\mathbf{8 2 . 0 2}$ \\
\hline $\mathbf{8}$ & $4.254,05$ & 30 & $\mathbf{1 4 1 . 8}$ & & 982,67 & 12 & $\mathbf{8 1 . 8 9}$ \\
\hline $\mathbf{9}$ & $4.478,69$ & 19 & $\mathbf{2 3 5 . 7 2}$ & & $1.099,86$ & 13 & $\mathbf{8 4 . 6 1}$ \\
\hline $\mathbf{1 0}$ & $4.202,40$ & 15 & $\mathbf{2 8 0 . 1 6}$ & & 0,00 & 0 & $\mathbf{0}$ \\
\hline
\end{tabular}

TABLA 5

DISTRIBUCIÓN DE LOS PRODUCTOS DE LAS ÁREAS VASCULARES TOTALES $\left(\mu \mathrm{m}^{2}\right)$ POR EL NÚMERO DE VASOS SANGUÍNEOS

INTRATUMORALES, PARA CADA CASO DE TVT CANINO, SEGÚN FASE DE CRECIMIENTO TUMORAL

\begin{tabular}{ccc}
\hline & \multicolumn{2}{c}{ Fase crecimiento tumoral } \\
\cline { 2 - 3 } Caso & Progresivo & Regresivo \\
\hline $\mathbf{1}$ & 181.50 & 41.57 \\
\hline $\mathbf{2}$ & 412.17 & 186.01 \\
\hline $\mathbf{3}$ & 199.73 & 14.88 \\
\hline $\mathbf{4}$ & 185.64 & 32.03 \\
\hline $\mathbf{5}$ & 201.93 & 188.1 \\
\hline $\mathbf{6}$ & 249.13 & 51.62 \\
\hline $\mathbf{7}$ & 93.61 & 82.02 \\
\hline $\mathbf{8}$ & 141.8 & 81.89 \\
\hline $\mathbf{9}$ & 235.72 & 84.61 \\
\hline $\mathbf{1 0}$ & 280.16 & 0 \\
\hline Promedio & $\mathbf{2 1 8 . 1 4 a}$ & $\mathbf{7 6 . 2 7 b}$ \\
\hline DS & $\mathbf{8 6 . 3 5}$ & $\mathbf{6 5 . 0 1}$ \\
\hline $\mathbf{y}$ & & \\
\hline
\end{tabular}

a y $b$ representan diferencias significativas $(p \leq 0.05)$.

distribución fueron histológicamente similares a lo observado previo al tratamiento, aunque estos no se encontraban distendidos y la presencia de glóbulos rojos en su interior fue escasa, coincidiendo con lo observado por Ramos (2004) y Jana (2005), que registraron, mediante técnicas inmunohistoquímicas, una disminución del área endotelial en muestras de TVT canino en fase regresiva. Estas características fueron concordantes con el estudio morfométrico del lecho vascular realizado en base a la presencia de eritrocitos en el estroma tumoral de los mismos casos, con tinción Papanicolau. En esta medición, la disminución del área ocupada por elementos eritrocitarios, fue estadísticamente significativa ( $\mathrm{p} \leq 0.05$ ).

La falta de dilatación demostrada por los vasos sanguíneos podría estar asociada al colapso del parénquima tumoral descrito por Mukaratirwa y Gruys (2004), en donde se describe el reemplazo del tejido tumoral por estroma fibroso. Este tejido ejercería un efecto mecánico, aumentando la presión sobre el lúmen de los vasos presentes, colapsándolos, lo que; esto además podría explicar en parte la disminución de glóbulos rojos presentes en los cortes regresivos, pues el colapso de los vasos dificultaría la irrigación sanguínea de la masa tumoral.

\section{FIGURA 6}

PRODUCTOS DE LAS ÁREAS VASCULARES TOTALES $\left(\mu \mathrm{m}^{2}\right)$ POR EL NÚMERO DE VASOS SANGUÍNEOS INTRATUMORALES

PARA CADA CASO DE TVT CANINO, SEGÚN FASE DE CRECIMIENTO TUMORAL

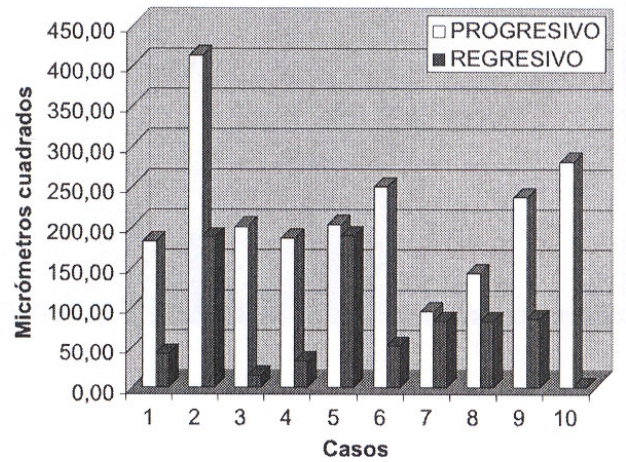


Por otro lado, el efecto del tratamiento con VCR también podría estar relacionado con la disminución de células eritrocitarias en el tumor, debido a que la presencia de eritrocitos está directamente relacionada con la existencia de vasos sanguíneos funcionales que sean capaces de transportarlos hacia los tejidos tumorales, con el fin de suplementar los altos requerimientos metabólicos que exiben las células neoplásicas (Folkman y Shin, 1992). Así, en los estudios realizados por Ramos (2004) y Jana (2005) con Dexametasona, ésta se describe como un agente antiangiogénico que afecta directamente la formación de nueva vasculatura tumoral, a través de la inhibición de la angiogénesis tumoral (Folkman e Ingber, 1987) y del efecto pro-apoptótico de la dexametasona sobre las células endoteliales (Lansink et al., 1998), lo que habría impedido la llegada de eritrocitos al estroma tumoral. Del mismo modo, se ha descrito en la literatura un efecto antivascular relacionado a la VCR y a los agentes antitubulínicos, mediante el cual estas drogas se unirían a los microtúbulos de las células endoteliales tumorales en proliferación, en forma selectiva, deteniendo el ciclo celular y promoviendo la apoptosis (Hayot et al., 2002; Thorpe et al., 2003; Bayless y Davis, 2004). Esta selectividad se debería a que generalmente, las células que conforman los vasos sanguíneos del resto del organismo permanecen quinescientes (detenidos en fase G0) y por lo tanto, no se dividen (Friedlander, 1995; Strauss, 2001).

Por otra parte, el efecto pro-apoptótico de la VCR sobre las células tumorales, descrito por Reyes (2004), también impediría la producción de importantes factores pro-angioénicos tumorales, como el factor de transcripción indudible por hipoxia (HIF-1) (Wang et al., 1995; Strauss, 2001) y el VEGF (Dong et al., 2004), bloqueando así el desarrollo de angiogénesis tumoral, como ha sido expuesto por Hanahan y Folkman (1996) y Jung et al. (2002).

En la fase regresiva del TVT inducida por tratamiento con VCR los vasos predominantes detectados mediante las técnicas de tinción histológicas ocupadas (Pap, H-E, vG y PAS), fueron principalmente de pequeño calibre, mientras que el número de vasos sanguíneos y el lecho vascular fueron notoriamente menores que en las muestras tomadas previo al tratamiento.

Para evaluar en forma más directa el efecto antivascular postulado para VCR se midió morfométricamente el área ocupada por los vasos sanguíneos del TVT, confirmando que existía una disminución estadísticamente significativa $(\mathrm{p} \leq 0.05)$, asociada a la fase regresiva turmoral inducida por el tratamiento con VCR. Esto, concuerda con el aspecto macroscópico que presentaron los tumores terminado el tratamiento, los que se observaron más pálidos y menos hemorrágicos que al inicio del tratamiento.

$\mathrm{Al}$ estudiar el tipo de vasos sanguíneos presentes en las diferentes fases tumorales se observó un predominio de vasos de menor tamaño en la fase regresiva. Esto no coincide con lo esperado para el efecto antivascular y antiangiogénico descrito para la VCR, debido a que la acción de las drogas antitubulínicas se produciría precisamente sobre los vasos de menor calibre, como capilares y vasos angiogénicos (Bayless y Davis, 2004), y no sobre los de mayor tamaño, que corresponderían a la población de vasos sanguíneos más maduros que son menos susceptibles al efecto antimitótico de las drogas antimicrotubulares (Hadfield et al., 2003). Además el bloqueo indirecto de la angiogénesis promovido por la destrucción las células tumorales (Hanahan y Folkman, 1996), también se fomentaría una disminución del recuento de vasos de pequeño calibre, contrario a lo detectado en el estudio morfométrico.

Esta discrepancia tal vez podría ser atribuída a que al no existir capilares ni neovasculatura para transportar el lecho vascular hacia el tumor, los vasos de mayor tamaño podrían estar colapsados, por lo que no habrían sido evaluados mediante las técnicas empleadas. Por otra parte, los vasos observados en los cortes histopatológicos de TVT regresivo podrían corresponder a vasos extratumorales provenientes de tejidos del huésped. Tras el colapso del parénquima tumoral descrito por Mukaratirwa y Gruys (2004) para la fase regresiva, se produciría una retracción de la masa tumoral, lo que podría promover la combinación de tejidos tumorales y del hospedero, generando la aparición de vasos sanguíneos extratumorales en las muestras regresivas. Asi mismo, en las muestras de tejido tumoral en fase regresiva solo se apreciaban escasos remanentes de tejido neoplásico con abundante tejido colagenoso e infiltración leucocitaria.

El análisis morfométrico se basó en la detección de vasos de lumen expandido y la presencia de eritrocitos dentro de ellos, por lo tanto no era posible detectar brotes endoteliales asociados a angiogénesis tumoral. Las técnicas ocupadas en este trabajo no son específicas para la detección de endotelio vascular, lo cual es posible a través del empleo de técnicas inmunohistoquímicas que detecten moléculas de expresión endotelial como el factor de vonWillebrand o CD31 (Weidner et al., 1992; Obermair et al., 1996). 
Para evaluar el efecto antiangiogénico del sulfato de Vincristina en el Tumor Venéro Transmisible canino (TVT), en el futuro se deberán diseñar experiencias que incluyan estas técnicas.

\section{REFERENCIAS}

ADAMS, E. W.; CARTER, L. P., SAPP, W. J. 1981. Cytogenetic observations on the canine venereal tumor in long-term culture. Cornell Vet 71(3): 336-346.

AOUDJT, F; VUORI, K. 2001. Integrin signaling inhibits paclitaxel-induced apoptosis in breast cancer cells. Oncogene 16; 20(36): 4995-5004.

BAYLESS, K. J.; DAVIS, G. E. 2004. Microtubule Depolymerization Rapidly Collapses Capillary Tube Networks in Vitro and Angiogenic Vessels in Vivo through the Small GTPase Rho. J Biol Chem 279(12): 11686-11695.

Bellhoussine, R.; Morjani, H.; Gillet, R.; Palissot, V.; MANFAIT, M. 1999. Two distinct modes of oncoprotein expression during apoptosis resistance in vincristine and daunorrubicin multidrug-resistant HL60 cells. Adv Exp Med Biol 457: 365-81.

Bootri, M. J. 1994. Canine Transmissible Venereal and Ovarian Papillary Cystoadeno-carcinoma in a Bitch. J Small Anim Pract 16: 393-398.

Brown, N. O.; Calvert C.; Macewen, E. G. 1980. Chemotherapeutic management of transmissible venereal tumor in 30 dogs. JAVMA 176: 983-986.

Dong, J.; Grunstein, J.; TeJada, M.; PEAle, F; Frantz, G.; LianG, W.; BAI, W.; YU, L.; KOWAL SKI, J.; LIANG, X.; FuH, G.; GERBER, H. P.; FERRARA, N. 2004. VEGF-null cells require PDGFR signaling-mediated stromal fibroblast recruitment for tumorigenesis. EMBO J 23(14): 2800-2810.

DUARTE, M. 2002. Indicadores de Proliferación celular en Tumor Venéreo Transmisible Canino. Memoria Título Médico Veterinario. Santiago, Chile. Universidad de Chile, Facultad de Ciencias Veterinarias y Pecuarias. 27-29.

FOLKMAN, J.; INGBER, D. 1987. Angiostatic steroids: method of discovery and mechanism of action. An Surg 206: 374-383

FolkMAN, J.; SHIN, Y. 1992. Angiogenesis. J Biol Chem 267(16): 10931-10934

FRIEDLANDER, M. A.; BROOKS, P. C.; SHAFFER, R. WV. 1995. Definition of two angogenic pathways by distinct alfa $v$ integrins. Sci 270: 1500-1502.

GONZÁLEZ, C. M.; GRIFFey, S. M.; NAYdAN, D. K.; FloRes, E.; Cepeda, R.; Cattaneo,g.; Madewell, B. R. 2000. Canine Transmisible Venereal Tumour: a Morphological and Inmunohistochemical Study of 11 Tumours in Growth Phase and during Regresión alter Chemotherapy. J Comp Pat 122: 241-248.

GonzÁlez, C. M.; Cepeda, R.; Flores, E.; Cattaneo, G.; ColARTE, K. 2002. Separación y purificación de células de tumor venéreo transmisible canino espontáneo, en gradiente de densidad de polisucrosa y diatrizoato de sodio. Av Cs Vet 17: 18-23.

GronINGER, E.; MEEUTwSEN-DE BOER, G. J.; DE GRAAF, S. S. N.; KAMPS W. A.; DE BONT, E. S. J. M. 2002. Vincristine induced apoptosis in acute lymphoblastic eukaemia cells: A mitochondrial controlled pathway egulated by reactive oxygen species? Inter J Oncol 21: 1339-1345.
HADFIELd, J. A.; DUCKI, S.; Hirst, N.; MCGOWN, A. T. 2003. Tubulin and microtubules as targets for anticancer drugs. Prog Cell Cycle Res 32 (5): 309-325.

HaNAHAN, D.; Folkman, J. 1996. Patterns and emerging mechanisms of the angiogenic switch during tumorigenesis. Cell 86: 353-364.

Hayot, C.; Farinelle, S.; De Decker, R.; Decaestecker, C.; DARRo, F.; KISS, R.; VAN DAMME, M. 2002. In vitro pharmacological characterizations of the anti-angiogenic and anti-tumor cell migration properties mediated by microtubuleaffecting drugs, with special emphasis on the organization of the actin cytoskeleton. Internat J Oncol 21: 417-425.

JANA, K. 2005. Proliferacion celular en tumor venéreo transmisible canino tratado con dexametasona. Memoria Título Médico Veterinario. Santiago, Chile. U. Chile, Fac. de Cs. Veterinaria y Pecuarias. $58 \mathrm{pp}$.

Jeglum, K. 1994. Principios de la Terapia Anticancerosa. En: Villouta, G.; Gonzáles, C. Compendio del Curso Internacional de Oncología Veterinaria. U. Chile, Fac. Cs. Veterinarias y Pecuarias, Escuela de Postgrado, Dpto. Patología Animal. Santiago, Chile. pp: 26-30.

JORDAN, M. A.; WIL.SON, L. 2004. Microtubules as a target for anticancer drugs. Nature Reviews. Cancer 4: 253-266

JunG, Y. D.; AhMad, S. A.; LiU, W.: ReinMuth, N.: PARIKH, A.; STOELTZing, O.; FAN, F; Ellis, L. M. 2002. The role of the microenvironment and intercellular cross-talk in tumor angiogenesis. Semin Cancer Biol 12: 105-112.

LANSINK, M.; KOOLWIJK, P.; VAN Hinsbergh, V.; KoOISTRA, T. 1998. Effects of Steroid Hormones and Retinoids on the Formation of Capillary-like Tubular Structures of Human Microvascular Endothelial Cells in Fibrin Matrices is Related to Urokinase Expression. Blood 92(3): 927-938.

LOMBARDI C. 1995. Respuesta Inmonológica de TVT canino en Relación al Tratamiento Quimioterapeutico. Tesis Magister, Fac. de Cs. Veterinarias y Pecuaria U. de Chile. pág: 130-136.

LÓPEZ, M.; LeygThon, C.; GRAF, M. 1985. Técnicas de Laboratorio para Citología e Histopatología. $2^{\text {a }}$ edición. U. de Chile Fac. de Medicina, Dpto. de Biología celular y Genética. Pp: 104-111.

Mukaratirwa, S.; Gruys, E. 2004. Canine transmissible venereal tumour: cytogenetic origin, immunophenotype, and immunobiology. Vet Q 25: 101-111.

Natsume, T.; Watanabe, J.; Koh, Y.; Funo, N.; Ohe, Y.; HorIUCHI T.; SAIJO, N.; NISHIO, K.; KOBAYASHI M. 2003. Antitumor activity of TZT-1027 (Soblidotin) against vascular endotelial growth factor-secreting human lung cancer in vivo. Cancer Sci 94: 826-833.

OBERMAIR, A.; WANNER, C.; BILgi, S.; SPEISER, P.; KAIDER, A.; Reinthaller, A.; Leodolter, S.; Gitsch, G. 1996. Tumor Angiogenesis in stage IB cervical cancer: Correlation of microvessel density With Survival. Am J Obstet Gynecol 1996: 126-131.

RAMOS, R. 2004. Actividad anti-angiogénica de la dexametasona en tumor venéreo transmisible canino. Memoria Título Médico Veterinario. Santiago, Chile. U. Chile, Fac. de Cs. Veterinaria y Pecuarias. Pp: 27-36.

REYES, S. 2004. Apoptosis en Tumor Venéreo Transmisible Canino, durante fase progresiva y regresiva. Memoria Título Médico Veterinario. Santiago, Chile. U. Chile, Fac. de Cs. Veterinaria y Pecuarias. 27-29.

RoGERS, K. S. 1997. Transmisible venereal tumor. The Compendium of Contuining Education 19: 1036-1045. 
Seki, K.; Yoshikawa, H.; Shiki, K.; Hamada, Y.; Akamatsu, N.; TASAKA, K. 2000. Cisplatin (CDDP) specifically induces apoptosis via secuencial activaction of caspasa-8, -3 and -6 in osteosarcoma. Cancer Chemoter Pharmacol 45(3): 199-206

SNEDECOR, G.; COCHRAN, W. 1980. Statistical Method. Edit. Iowa State University Press. P: 290

SrivastaVa, R. K.; SRIVASTAVA, A. R.; Korsmeyer, S. J.; NesteROVA M.; CHO-CHUNG, Y. S.; LONGO, D. L. 1998. Involvement of Microtubules in the Regulation of Bcl2 Phosphorylation and Apoptosis through Cyclic AMP-Dependent Protein Kinase. Molec Cell Biol: 3509-3517.

STRAUSS L. 2001 Estudio de la actividad angiogénica y antimetastásica de la betametasona sola asociada a beta ciclodextrina sulfatada sobre tumores malignos. Proyecto para optar al grado de licenciado en medicina veterinaria y al Título Profesional de Médico Veterinario. Santiago, Chile. Fac. de Cs Silvoagropec. Escuela de Med. Vet. Universidad Mayor. $158 \mathrm{Pp}$.

Tella, M.; Ajalla, O.; TAIWO, V. 2004. Complete regresion of Transmissible Venereal Tumour (TVT) in Nigerian mongrel dogs with Vincristine sulphate chemotherapy. African J Biomed Res 7 (3): 133-138.

Thorpe, P. E.; Chaplin, D. J.; Blakey, D. C. 2003. The First International Conference on Vascular Targeting: Meeting Overview. Cancer Res 63: 1144-1147

TORRES, C. 1999. Aspectos histopatológicos e Inmunohistoquímicos del Tumor Venéreo Transmisible Canino, en Progresión y Regresión Mediante Análisis de Imagen. Memoria de Título Médico Veterinario. Santiago, Chile. U. De Chile, Fac. De Cs. Vet. y Pec. 64 Pp.

WANG, G. L.; JiAnG, B. H.; RuE, E. A., SEMENAZA, G. L. 1995. Demostration that mutation of the type II transforming growth beta receptor inactivates its tumour suppressor activity in replication error-positive colon carcinoma cells. Proc Nat Acad Sci 92: 5510-5514.

WEIDNER, N.; FOLKMAN, J.; POZZA, F.; BEVILACQUA, P.; ALFRED, E. N.; Moore, D. H.; Meli, S.; Gasparini, G. 1992. Tumor angiogenesis : a new significant and independent prognostic indicator in early-stage breast carcinoma. J Nat Can Inst 84 : 1875-1887. 